\title{
Classification of cancer cell lines using matrix-assisted laser desorption/ionization time-of-flight mass spectrometry and statistical analysis
}

\author{
VLAD SERAFIM $^{1,2}$, AJIT SHAH ${ }^{2}$, MARIA PUIU $^{1}$, NICOLETA ANDREESCU $^{1}$, \\ DORINA CORICOVAC ${ }^{3}$, ALEXANDER E. NOSYREV $^{4}$, DEMETRIOS A. SPANDIDOS ${ }^{5}$, \\ ARISTIDES M. TSATSAKIS ${ }^{6}$, CRISTINA DEHELEAN ${ }^{3}$ and IULIA PINZARU ${ }^{3}$
}

\begin{abstract}
${ }^{1}$ Center of Genomic Medicine, 'Victor Babes' University of Medicine and Pharmacy, Timisoara 300041, Romania;
${ }^{2}$ Department of Natural Sciences, Middlesex University, London NW4 4BT, UK; ${ }^{3}$ Department of Toxicology,

Faculty of Pharmacy, 'Victor Babes' University of Medicine and Pharmacy, 300041 Timisoara, Romania;

${ }^{4}$ Central Chemical Laboratory of Toxicology, I.M. Sechenov First Moscow State Medical University, 119991 Moscow,

Russia; ${ }^{5}$ Laboratory of Clinical Virology, Medical School; ${ }^{6}$ Department of Forensic Sciences and Toxicology,

Faculty of Medicine, University of Crete, 71003 Heraklion, Greece
\end{abstract}

Received February 2, 2017; Accepted July 12, 2017

DOI: $10.3892 /$ ijmm.2017.3083

\begin{abstract}
Over the past decade, matrix-assisted laser desorption/ ionization time-of-flight mass spectrometry (MALDI-TOF MS) has been established as a valuable platform for microbial identification, and it is also frequently applied in biology and clinical studies to identify new markers expressed in pathological conditions. The aim of the present study was to assess the potential of using this approach for the classification of cancer cell lines as a quantifiable method for the proteomic profiling of cellular organelles. Intact protein extracts isolated from different tumor cell lines (human and murine) were analyzed using MALDI-TOF MS and the obtained mass lists were processed using principle component analysis (PCA) within Bruker Biotyper ${ }^{\circledR}$ software. Furthermore, reference spectra were created for each cell line and were used for classification. Based on the intact protein profiles, we were able to differentiate and classify six cancer cell lines: two murine melanoma (B16-F0 and B164A5), one human melanoma (A375), two human breast carcinoma (MCF7 and MDA-MB-231) and one human liver carcinoma (HepG2). The cell lines were classified according to cancer type and the species they originated from, as well as by their metastatic potential, offering the possibility to differentiate non-invasive from invasive cells. The obtained results pave the
\end{abstract}

Correspondence to: Dr Dorina Coricovac, Department of Toxicology, Faculty of Pharmacy, 'Victor Babes' University of Medicine and Pharmacy, 2 Eftimie Murgu Sq, 300041 Timisoara, Romania E-mail: dorinacoricovac@umft.ro

Key words: cancer cell lines, matrix-assisted laser desorption/ ionization time-of-flight mass spectrometry, principle component analysis, reference spectra library, biotyping, intact protein profiling way for developing a broad-based strategy for the identification and classification of cancer cells.

\section{Introduction}

The development and application of matrix-assisted laser desorption/ionization time-of-flight mass spectrometry (MALDI-TOF MS) for the identification of microorganisms has been revolutionary. Microbial proteomics has attracted attention worldwide and this has resulted in the development of novel identification methods which are rapid, robust and relatively inexpensive (1).

For the identification of microorganisms, MALDI-TOF MS analysis typically concentrates on intact proteins with a molecular weight in the range of $2-20 \mathrm{kDa}$. The proteins in this range are mainly ribosomal proteins which represent approximately $30 \%$ of the total proteins from a microbial cell (2). Following mass spectral analysis, the raw spectra are processed using a peak recognition algorithm. The data generated following MALDI-TOF MS analysis consist of two large sets of values: mass-to-charge $(\mathrm{m} / \mathrm{z})$ and intensity. Each $\mathrm{m} / \mathrm{z}$, value has a corresponding intensity value. The signal extraction, also known as peak picking, is often corrupted by noise. Therefore, various algorithms have been developed for obtaining peaks that correspond to true peptide/proteins signals (3). This is a very important step in data analysis as different peak recognition algorithms may have a considerable effect on the peak list, and therefore should be adjusted with care (4). Once extracted, the peak lists are compared to a dedicated database which contains reference mass spectra of known microbial strains. The first such platform 'MALDI Biotyper' was developed by Bruker Daltonics. Another platform combines the Shimadzu mass instrumentation and software 'Launchpad' with a centralized database 'SARAMIS' provided by BioMerieux (Marcy l'Etoile, France) (1,5). 
Although mainly used for microbial identification, intact protein profiles have also been used successfully for the characterization and identification of mammalian cell lines (6-8). Karger et al (6) identified 66 cell lines from 34 species using reference spectra library created by MALDI Biotyper, while Povey et al (8) used partial least squares discriminant analysis model to predict the phenotype of recombinant mammalian cell lines.

The methods currently used for the identification and characterization of cancer cells, namely DNA fingerprinting, immunohistochemistry and flow cytometry (9-12), require specific reagents which limit the degree of multiplexing. In addition, these methods require laborious sample preparation which leads to an increased analysis time. The proteomic approach can achieve a level of multiplexing where several cell lines can be analyzed without changing the method parameters and without using specialized materials and reagents. The aim of this study was to assess the potential of using MALDI-TOF MS for the classification of cancer cell lines. To achieve this, the procedure for the taxonomic classification of microorganisms was adapted. Six cancer cell lines (murine and human) were used in this study: B16-F0 and B164A5 (murine melanoma cells), A375 (human melanoma), HepG2 (human liver carcinoma), MCF7 (human breast carcinoma) and MDA-MB-231 (human breast carcinoma). The statistical analysis was processed using MALDI Biotyper software. These data were used for a better observation of differences regarding the species and metastatic potential, differences well-defined between the two human breast carcinoma cell lines (MCF7 and MDA-MB-231) and two murine melanoma cell lines (B16-F0 and B164A5). As an end point, a different cell line was applied for an upgraded picture: HepG2 (human liver carcinoma).

\section{Materials and methods}

Cell lines and reagents. The cancer cell lines used in the present study, B16-F0 [murine melanoma; CRL-6322 $2^{\mathrm{TM}}$, American Type Culture Collection (ATCC), Manassas, VA, USA], B16 melanoma 4A5 (murine melanoma; 94042254; Sigma-Aldrich Chemie GmbH, Munich, Germany), A375 (human melanoma; CRL-1619 ${ }^{\mathrm{Tm}}$; ATCC), HepG2 (human liver carcinoma; HB8065 ${ }^{\mathrm{TM}}$; ATCC), MCF7 (human breast carcinoma; HTB22 ${ }^{\mathrm{TM}}$; ATCC) and MDA-MB-231 (human breast carcinoma; HTB26 ${ }^{\mathrm{TM}}$; ATCC), were acquired from Sigma-Aldrich Chemie $\mathrm{GmbH}$ and ATCC as frozen items.

The specific reagents for cell culture [Dulbecco's modified Eagle's medium (DMEM); Eagle's Minimum Essential Medium (EMEM)], fetal bovine serum (FBS), antibiotic mixture of penicillin/streptomycin, phosphate-buffered saline (PBS), Trypsin/EDTA and trypan blue were acquired from Sigma-Aldrich Chemie GmbH and ATCC.

Ethanol, formic acid, trifluoroacetic acid and acetonitrile were acquired from Sigma-Aldrich Chemie $\mathrm{GmbH}$. $\alpha$-cyano-4-hydroxycinnamic acid (Bruker HCCA matrix) and protein I calibration standard were acquired from Bruker Daltonics (Bremen, Germany).

Cell culture. The murine melanoma (B164A5 and B16-F0), human melanoma (A375) and human breast carcinoma (MDA-MB-231) cell lines were cultured in DMEM with $4.5 \mathrm{~g} / 1$ glucose, $2 \mathrm{mM}$ L-glutamine and supplemented with 10\% FBS and antibiotic mixture $(100 \mathrm{U} / \mathrm{ml}$ penicillin and $100 \mu \mathrm{g} / \mathrm{ml}$ streptomycin). The human liver carcinoma (HepG2) and human breast carcinoma (MCF7) were cultured in EMEM, supplemented with $10 \%$ FBS and antibiotic mixture. The cells were kept in standard conditions as follows: a humidified atmosphere with $5 \% \mathrm{CO}_{2}$ at a temperature of $37^{\circ} \mathrm{C}$ and were passaged every 2-3 days.

Scratch assay. The migratory character of the tumor cells used in this study was examined by the means of a scratch assay. In brief, $2 \times 10^{5}$ cells/well were seeded in 12 -well plates in specific culture medium and when the confluence was appropriate $(85-90 \%)$ a gap/scratch was drawn in the middle of the well with a $10 \mu \mathrm{l}$ tip (13). The capacity of the cells to migrate and fill the gap was monitored for $24 \mathrm{~h}$ by acquiring images at different time points, namely 0,3 and $24 \mathrm{~h}$ using an Optika Microscopes Optikam Pro Cool 5 and Optika View (Optika, Ponteranica, Italy).

Protein extraction for MALDI-TOF MS analysis. Sample preparation for MALDI-TOF MS analysis was performed as follows: the culture medium was discarded from the flasks; the cells were washed with $10 \mathrm{ml}$ PBS and were subsequently incubated with $3 \mathrm{ml} \mathrm{0.025 \%} \mathrm{trypsin/EDTA} \mathrm{for} \mathrm{3-5} \mathrm{min.} \mathrm{The} \mathrm{reaction}$ was terminated by the addition of $10 \mathrm{ml}$ cell culture medium. The cells were stained with trypan blue and the cell number was established using the Neubauer cell counting chamber. The suspension was subsequently centrifuged at $1,700 \mathrm{x} \mathrm{g}$ for $5 \mathrm{~min}$. The supernatant was discarded and the cell pellet was mixed with $1 \mathrm{ml}$ of ethanol. Following centrifugation at $1,700 \mathrm{x} \mathrm{g}$ for $5 \mathrm{~min}$, the ethanol was removed and the pellet was reconstituted in $70 \%$ formic acid at a ratio of $20 \mu 1 / 1 \times 10^{6}$ cells. The mixture was left at room temperature for $2 \mathrm{~min}$, and then an equal volume of acetonitrile was added. The samples were then centrifuged at $10,000 \mathrm{x}$ g for $5 \mathrm{~min}$. An aliquot $(1 \mu \mathrm{l})$ of the supernatant was spotted in duplicate onto the MTP 384 ground steel MALDI target plate (Bruker Daltonics). The sample was allowed to dry at ambient temperature and then overlaid with $1 \mu \mathrm{l}$ of $\mathrm{HCCA}(5 \mathrm{mg} / \mathrm{ml}$ in a mixture of acetonitrile, water and trifluoroacetic acid 50:47.5:2.5\% v/v).

MALDI-TOF MS analysis. The MALDI-TOF MS instrument (Bruker Daltonics) was calibrated in a positive ion linear mode in a mass range of 5,000-20,000 m/z, using Bruker Protein Calibration Standard I containing insulin, ubiquitin I, cytochrome $c$ and myoglobin. Flex Control ${ }^{\circledR}$ (version 3.4) software was used to acquire the data and set the method parameters. The following settings were used: laser frequency, 2,000 Hz; smartbeam, '4_large'; sample rate and digitiser settings, $1.25 \mathrm{GS} / \mathrm{sec}$; accelerator voltage, $20.07 \mathrm{~kW}$; extraction voltage, $18.87 \mathrm{~kW}$; lens voltage, $5.58 \mathrm{~kW}$; and delayed extraction, $250 \mathrm{nsec}$. The laser intensity was adjusted such that the highest peak in the spectrum was in the range of $10^{4}$ arbitrary units. A 1,000 laser shots were used for each individual spectrum and minimum of 10 individual spectra (10,000 laser shots) were cumulated and saved.

Mass spectra processing. Mass spectra were processed using MALDI Biotyper Offline Client ${ }^{\circledR}$ software (version 3.1; Bruker Daltonics). The MALDI-TOF MS spectra were analyzed using principle component analysis (PCA). The following parameters 
were used for pre-processing the spectra: mass adjustment from 5,000 to $20,000 \mathrm{~m} / \mathrm{z}$; resolution, 1 ; processing method, spectra compressing; compressing factor, 10; smoothing method, 'Savitsky-Golay' with a frame size of $25 \mathrm{kDa}$; baseline subtraction method, multipolygon with a search window of $5 \mathrm{kDa}$ in 2 runs; spectra normalization, maximum; peak piking method, 'Local maximum' with 200 maximum peaks, signal-to-noise threshold, 0.01 and window width, $25 \mathrm{kDa}$. The PCA creation parameters were set as follows: mass from 9,500-12,500 $\mathrm{m} / \mathrm{z}$; resolution, 2 and number of principal components set to automatic.

For creating main spectra (MSP) the pre-processing parameters used were the same as those described for PCA analysis except that the mass range was 9,500 to $12,500 \mathrm{~m} / \mathrm{z}$. For the MSP creation method, the following parameters were used: maximum mass errors of each single spectrum, 2,000; desired mass error for the MSP, 200; desired peak frequency minimum, 25\%; maximum desired peak number for the MSP, 10. For the identification method, the following parameters were used: frequency threshold for spectra adjusting, 50; frequency threshold for score calculation, 5; maximum mass error of raw spectrum, 2,000; desired mass tolerance of the adjusted spectrum, 250; furthermore accepted mass tolerance of a peak 600 , parameter of the intensity correction function, 0.25. For creating the MSP dendrogram, the following parameters were used: distance measure, correlation; linkage, average; score threshold value for a single organism, 300; and score threshold value for a related organism, 0 .

\section{Results}

Characteristics of tumor cell appearance in culture. The cell lines employed in the present study in order to be classified and differentiated at proteomic level by the means of MALDI-TOF MS are illustrated in Fig. 1. It can be observed that the cells have different shapes, even though they originate from the same species [Fig. 1C (B16-F0) and D (B164A5) murine melanoma] or cancer type [Fig. 1E (MCF7) and F (MDA-MB-231) human breast carcinoma]. A375 (Fig. 1A) are human amelanotic cells with an epithelial morphology, whereas B16-F0 and B164A5 (murine) are melanin producing cells with a mixture of spindle-shaped and epithelial-like cells (B16-F0) or a fibroblast-like morphology (B164A5) (Fig. 1C and D, granules of melanin can also be observed).

The human liver carcinoma cell line, HepG2, has an epithelial morphology and the cells are strongly bonded (Fig. 1B). In the case of the two breast human carcinoma cell lines, MCF7 and MDA-MB-231, some differences were observed in terms of shape (an elongated shape in the case of MDA-MB-231 and a more round one in the case of MCF7 cells), albeit both are described to have an epithelial morphology (Fig. 1E and F).

To delineate the differences regarding the migratory character of the tumor cells studied, we performed a wound healing assay/scratch assay. As shown in Fig. 2, the A375 and HepG2 cells were not able to fill the gap drawn within $24 \mathrm{~h}$, what indicates a low migratory capacity.

B164A5 cells (murine melanoma) proved to be highly invasive, with the gap being completely filled after $24 \mathrm{~h}$. The B16-F0 cells also exhibited a potent migratory capacity, although to a lesser extent than the B164A5 cells (Fig. 3).
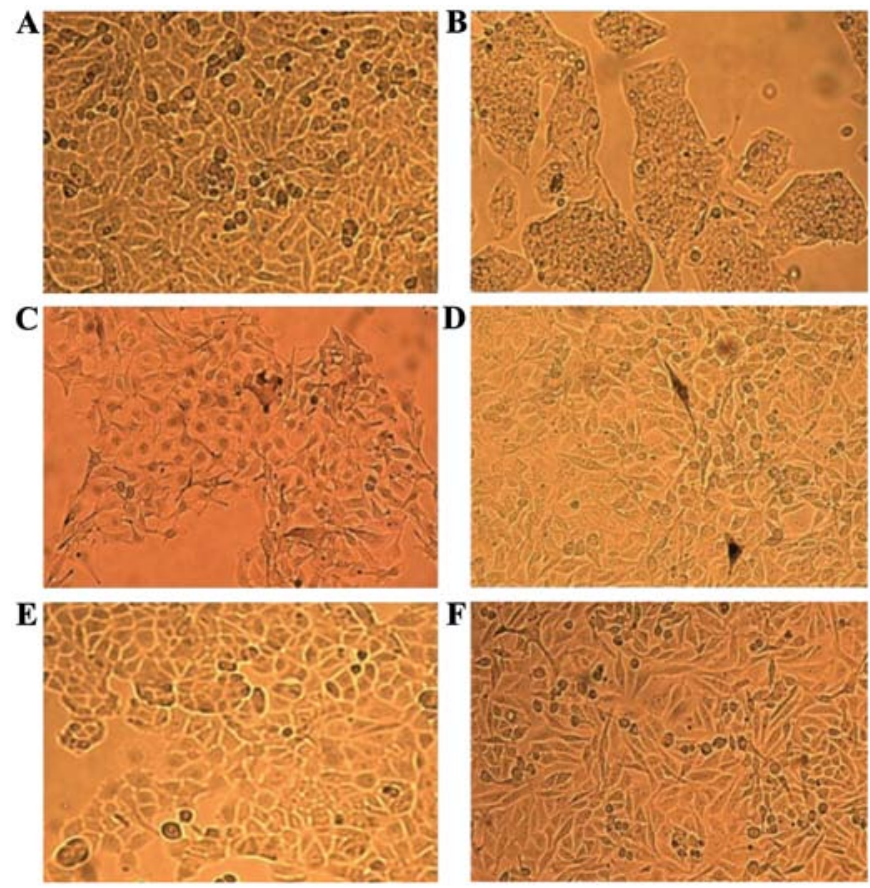

Figure 1. The appearance of tumor cells in culture. (A) A375 (human melanoma cells); (B) HepG2 (human liver carcinoma cells); (C) B16-F0 (murine melanoma cells); (D) B164A5 (murine melanoma cells); (E) MCF7 (breast carcinoma cells); and (F) MDA-MB-231 (breast carcinoma cells).

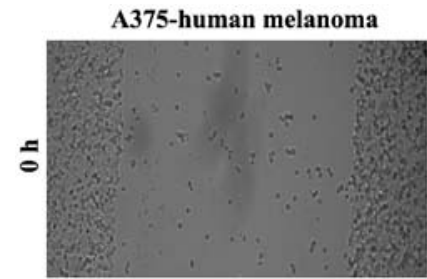

HepG2-human liver carcinoma
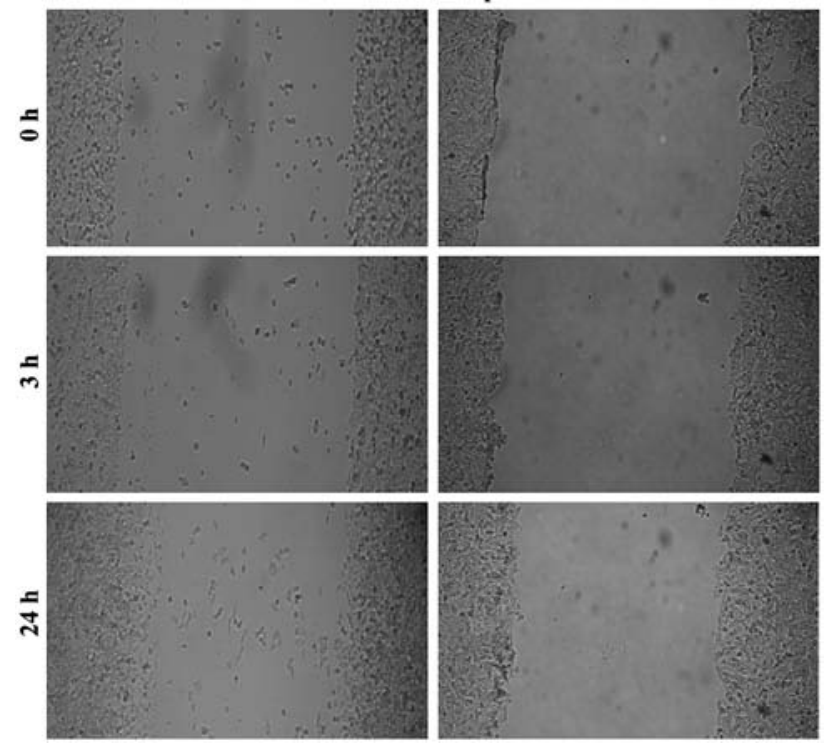

Figure 2. The migratory/invasive capacity of human melanoma cells (A375) and liver carcinoma cells (HepG2) over a period of $24 \mathrm{~h}$. The contrast of the images was increased for better visualization.

The comparison between the two breast carcinoma cell lines in terms of migratory capacity indicated that the MDA-MB-231 cells were more invasive (Fig. 4).

PCA analysis. PCA three-dimensional clustering (Fig. 5) revealed that the three human carcinoma cell lines (HepG2, MDA-MB-231 and MCF7) formed a distinct cluster, while the human (A375) and murine melanoma (B164A5 and B16-F0) cell lines formed another two separate clusters. 

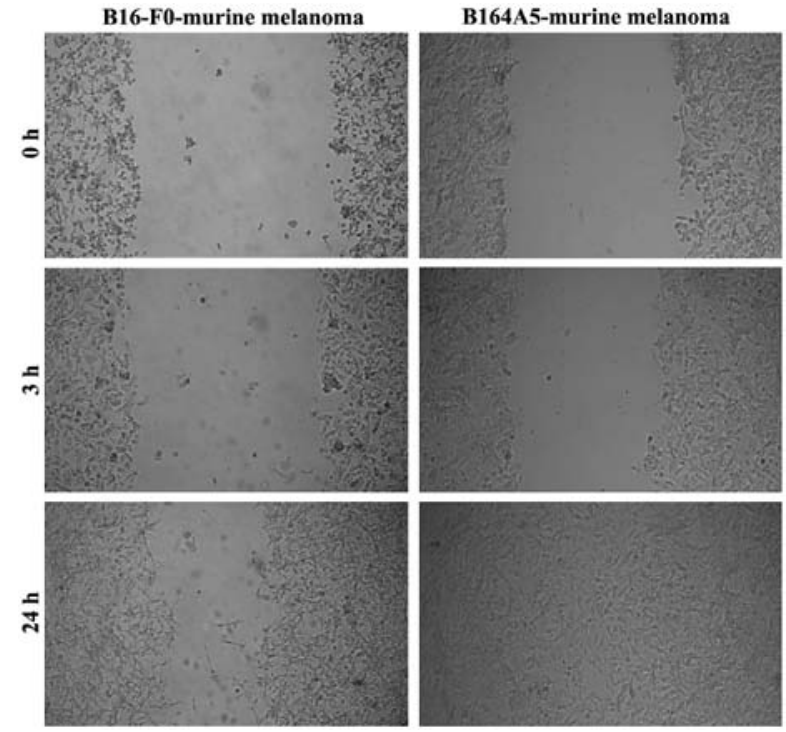

Figure 3. The migratory capacity of murine melanoma cells (B16-F0 and B164A5) over a period of $24 \mathrm{~h}$. The contrast of the images was increased for better visualization.

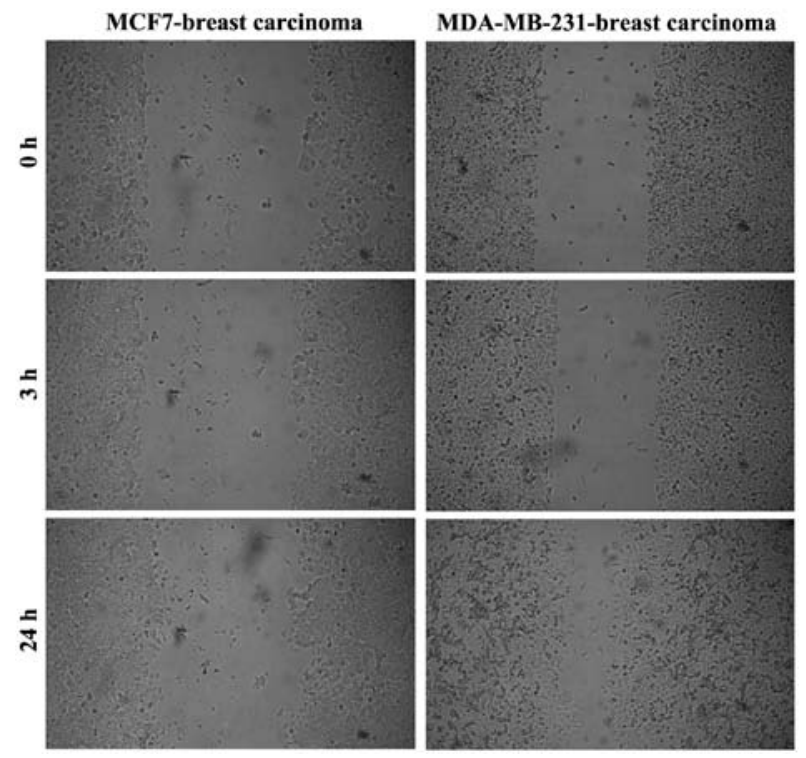

Figure 4 . The migratory capacity of human breast carcinoma cells (MCF7 and MDA-MB-231) over a period of $24 \mathrm{~h}$. The contrast of the images was increased for better visualization.

Within the human carcinoma cluster, and also in the PCA dendrogram (Fig. 6) the distance level that separated the breast carcinoma (MDA-MB-231) cell line from the liver carcinoma (HepG2) cell line was shorter than the distance level between the two breast carcinoma cell lines. The contribution of PC1, PC2 and PC3 was found to be 66,24 and $7 \%$, respectively (Fig. 7).

Creation and use of MSP. All MSP (Fig. 8) were used to confirm the classification of cell lines. This yielded a score for the level of similarity (Table I).

This was also illustrated as an MSP-based dendrogram (Fig. 9). A score value of 3 represents the maximum similarity, while 0 represent no relatedness. For individual

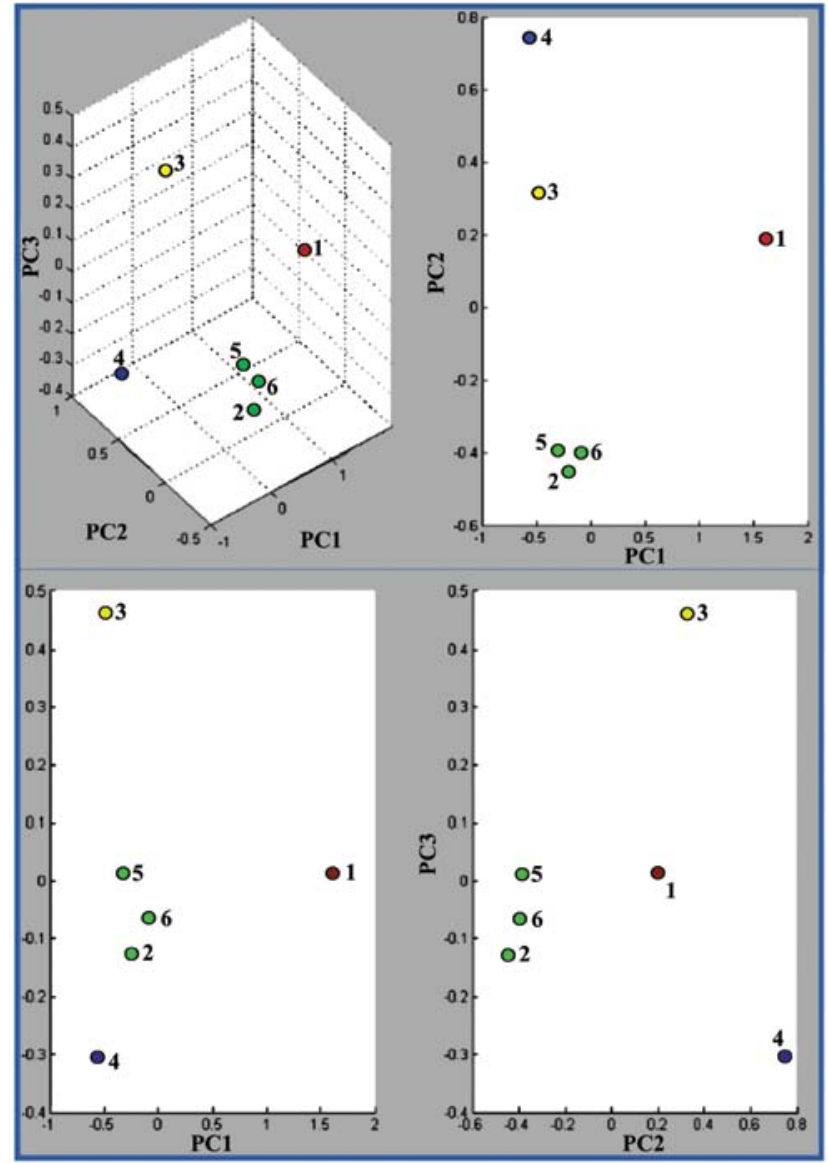

Figure 5. PCA spectra clustering using 9,500-12,500 $\mathrm{m} / \mathrm{z}$ interval. The top left score plot is a 3D plot of $\mathrm{PC} 1$ vs. $\mathrm{PC} 2$ vs. $\mathrm{PC} 3$ while on right is a $2 \mathrm{D}$ plots of $\mathrm{PC} 1$ vs. PC2, bottom left is $\mathrm{PC} 1$ vs. PC 3 and bottom right PC2 vs. PC3. Each dot represents one spectra: 1, A375 (human melanoma); 2, HepG2 (human liver carcinoma); 3, B16-F0 (murine melanoma); 4, B164A5 (murine melanoma); 5, MCF7 (human breast carcinoma); and 6, MDA-MB-231 (human breast carcinoma). PCA, principle component analysis.

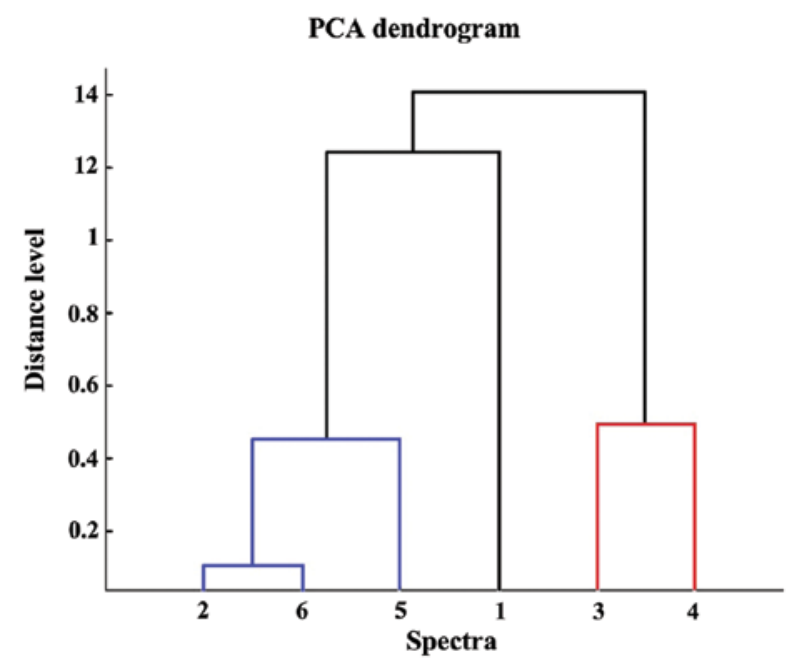

Figure 6. PCA dendogram. 1, A375 (human melanoma); 2, HepG2 (human liver carcinoma); 3, B16-F0 (murine melanoma); 4, B164A5 (murine melanoma); 5 , MCF7 (human breast carcinoma); and 6, MDA-MB-231 (human breast carcinoma). PCA, principle component analysis.

MSP, the results revealed a similarity between the same types of cancer. The only exception was the A375 MSP, where the 
Table I. The classification results obtained by comparing the MSP in rapport with each other.

\begin{tabular}{|c|c|c|c|}
\hline \multicolumn{2}{|c|}{ Individual MSP score } & \multicolumn{2}{|c|}{ Group MSP score } \\
\hline Cell line & Score & Cell group/type & Score \\
\hline \multicolumn{4}{|l|}{ A375 } \\
\hline A375 & 3 & Human melanoma & 3 \\
\hline MDA-MB-231 & 1.832 & Human carcinoma & 2.27 \\
\hline B 164A5 & 1.405 & Murine melanoma & 0.641 \\
\hline HepG2 & 1.397 & & \\
\hline B16-F0 & 1.389 & & \\
\hline MCF7 & 1.344 & & \\
\hline \multicolumn{4}{|l|}{ HepG2 } \\
\hline HepG2 & 3 & Human carcinoma & 2.505 \\
\hline MDA-MB-231 & 2.451 & Human melanoma & 1.397 \\
\hline MCF7 & 2.109 & Murine melanoma & 1.35 \\
\hline A375 & 1.397 & & \\
\hline B16-F0 & 1.386 & & \\
\hline B164A5 & 1.003 & & \\
\hline \multicolumn{4}{|l|}{ B16-F0 } \\
\hline B16-F0 & 3 & Murine melanoma & 2.782 \\
\hline B164A5 & 2.479 & Human carcinoma & 1.406 \\
\hline HepG2 & 1.498 & Human melanoma & 1.389 \\
\hline A375 & 1.389 & & \\
\hline MDA-MB-231 & 1.329 & & \\
\hline MCF7 & 1.304 & & \\
\hline \multicolumn{4}{|l|}{ B164A5 } \\
\hline B164A5 & 3 & Murine melanoma & 2.659 \\
\hline B16-F0 & 2.48 & Human melanoma & 1.405 \\
\hline A375 & 1.405 & Human carcinoma & $<0$ \\
\hline MCF7 & 1.354 & & \\
\hline HepG2 & 1.003 & & \\
\hline MDA-MB-231 & 0.641 & & \\
\hline \multicolumn{4}{|l|}{ MCF7 } \\
\hline MCF7 & 3 & Human carcinoma & 2.116 \\
\hline HepG2 & 2.109 & Human melanoma & 1.344 \\
\hline MDA-MB-231 & 1.464 & Murine melanoma & 1.331 \\
\hline B164A5 & 1.354 & & \\
\hline A375 & 1.344 & & \\
\hline B16-F0 & 1.304 & & \\
\hline \multicolumn{4}{|l|}{ MDA-MB-231 } \\
\hline MDA-MB-231 & 3 & Human carcinoma & 2.443 \\
\hline HepG2 & 2.449 & Human melanoma & 1.832 \\
\hline A375 & 1.832 & Murine melanoma & 0.574 \\
\hline MCF7 & 1.466 & & \\
\hline B164A5 & 1.393 & & \\
\hline B16-F0 & 1.343 & & \\
\hline
\end{tabular}

The maximum score is 3 . The higher the scores, the higher the relationship. MSP, main spectrum.

second hit was a breast carcinoma. In the MSP dendrogram however, all three human carcinoma cell lines (HepG2,

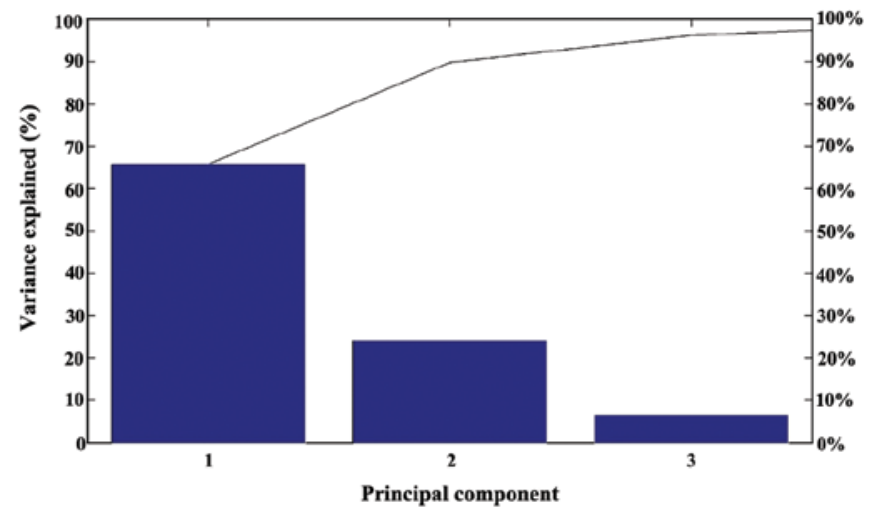

Figure 7. The contribution of three principal components to the PCA classification. The $\mathrm{PC} 1$ contribution was approximately $66 \%$ while that of $\mathrm{PC} 2$ and PC3 was approximately 24 and 7\%, respectively. PCA, principle component analysis.

MDA-MB-231 and MCF7) were grouped in a separate subclade from human carcinoma A375 cells. The murine melanoma cell lines (B164A5 and B16-F0) formed separate clades from all the human cells. In addition, within the human carcinoma clades, the distance level that separated the breast carcinoma cell line (MDA-MB-231) and the liver carcinoma cell line (HepG2) was shorter than the distance level between the two breast carcinoma cell lines (MCF7 and MDA-MB-231).

\section{Discussion}

MALDI mass spectrometry was firstly announced in the late 1980's by Hillenkamp and Karas (14) who developed a novel ionization technique that allowed the characterization of large biomolecules as proteins by mass spectrophotometer (15). Further improvements of the initial method propelled MALDI-TOF MS into the top of renowned tools for proteomic research. This technique is characterized by a high sensitivity, an ease of operation and automated capabilities, and can be used for the analysis of small organic or large biochemical compounds obtained from various sources $(15,16)$. Apart from its great usability in microbial identification, MALDI-TOF MS has proven to be a valuable cancer diagnostic tool, being applied to detect biomarkers for a plethora of cancer types, such as gastrointestinal cancer, lung cancer, renal and bladder cancer, ovarian cancer, leukemia (17), melanoma (18), breast carcinoma (19) and hepatic carcinoma (20).

The present study aimed to use the established Bruker Biotyper protocol with minimum modifications for the classification and differentiation of several human and murine cancer cell lines. The choice of HCCA matrix was also supported by evidence from a previous study on cell lines (6). The sample preparation process was very simple, rapid and inexpensive, and it was found that using $70 \%$ formic acid at a ratio of $20 \mu \mathrm{l}$ for $1 \times 10^{6}$ cells, provided the optimal spectra quality. Following the addition of acetonitrile, the final ratio was $0.025 \times 10^{6}$ cells $/ \mathrm{ml}$. A higher concentration of cells led to ion suppression, which resulted in an increased noise level and fewer assigned peaks, a fact previously reported by Povey et al (8). The formic acid extraction requires only $2 \mathrm{~min}$, a method that is faster compared with a previous report by Povey et al (8), 

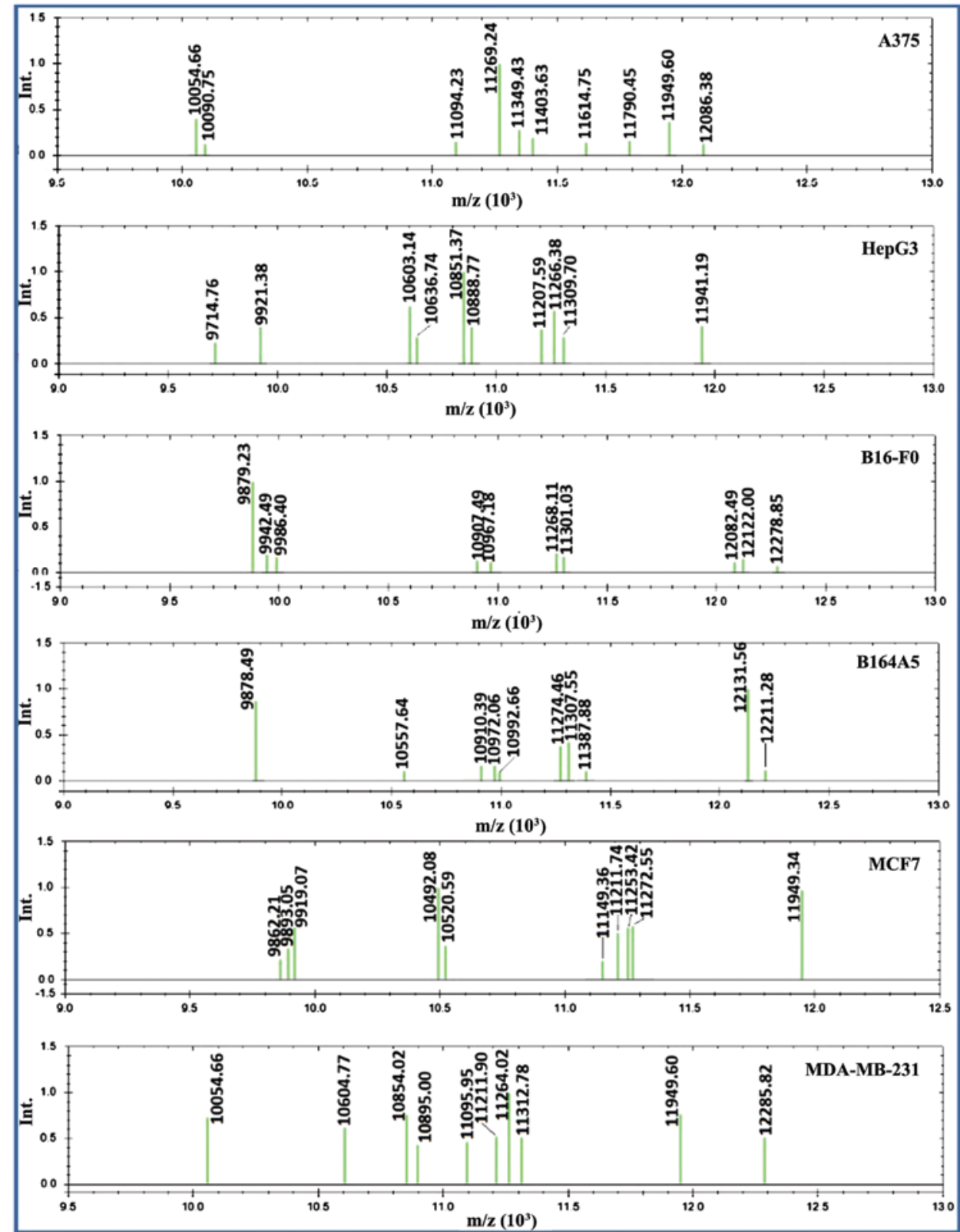

Figure 8. MSP spectra of the cancer cells. MSP were created using the 9,500-12,500 $\mathrm{m} / \mathrm{z}$ interval (Int). Each green bar represents one reference peak. Ten reference peaks were assigned for each reference spectrums. MSP, main spectra.

where a 4-h-long incubation with the matrix at $3^{\circ} \mathrm{C}$ was used. Analysis can therefore be performed using a reduced number of cells $(250,000$ cells). The analysis was performed in manual mode and the laser intensity was adjusted manually to obtain the highest signal-to-noise ratio. The method proved to have a very good shot-to-shot and spot-to-spot reproducibility correlated to the new application of this method in personalized medicine and tumor heterogeneity observation (21).

The most challenging step in this study was to establish a spectra processing method able to transform raw spectra (Fig. 10) into peak lists that, once processed using PCA algorithm, provided a realistic classification of the cell lines. This process involved three steps: smoothing, baseline subtraction and peak picking. For both smoothing and baseline subtraction the Bruker standard parameters were used. However, for peak picking the 'local maximum' method was used. This method is based on using the local maxima within

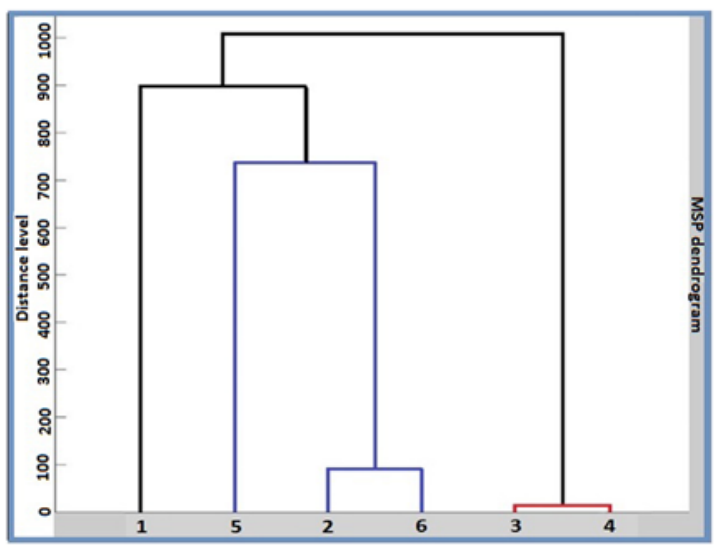

Figure 9. The MSP dendrogram. 1, A375 (human melanoma); 2, HepG2 (human liver carcinoma); 3, B16-F0 (murine melanoma); 4, B164A5 (murine melanoma); 5, MCF7 (human breast carcinoma); and 6, MDA-MB-231 (human breast carcinoma). MSP, main spectra. 


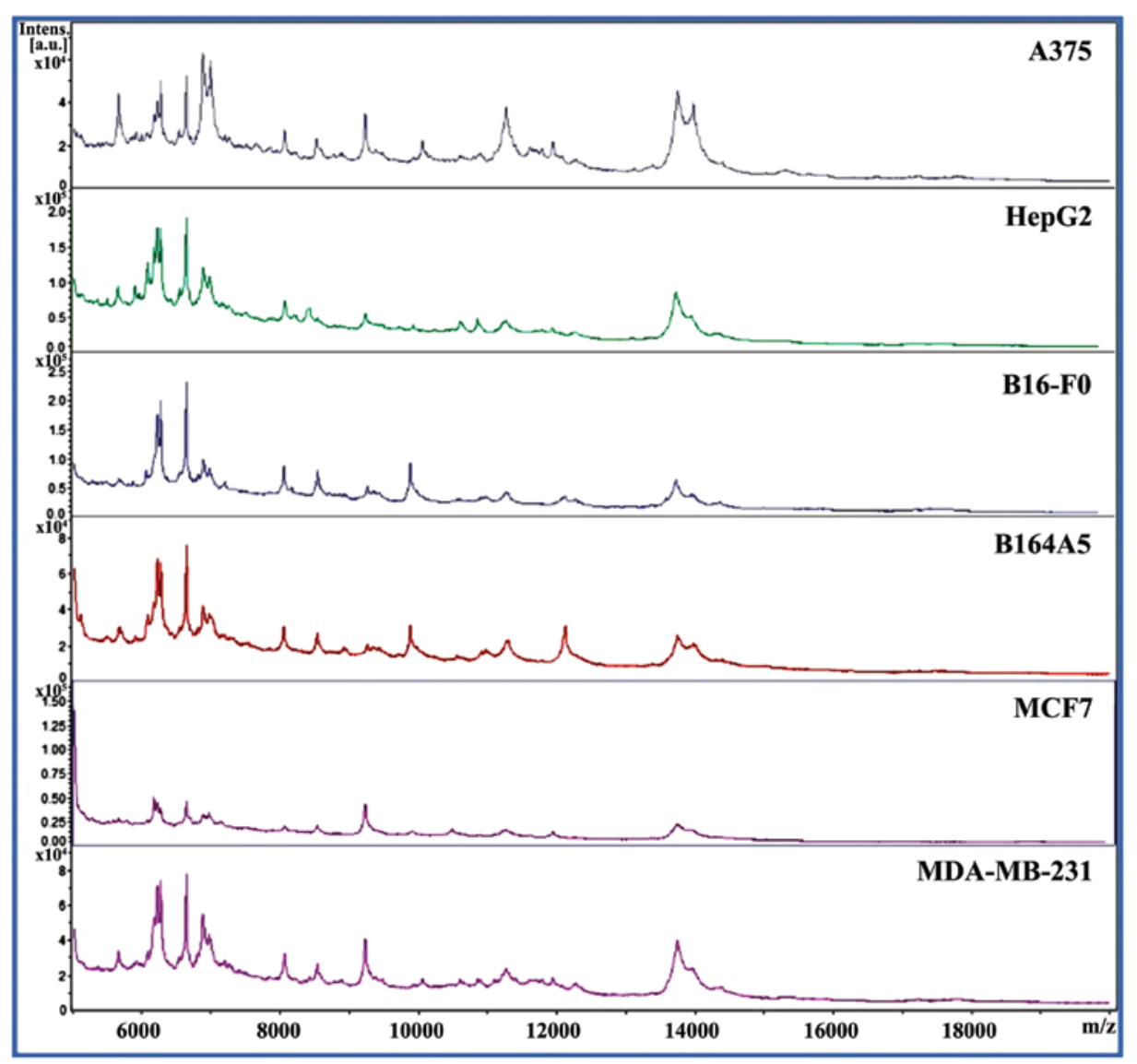

Figure 10. Raw spectra of the cancer cells: view that displays the entire acquisition range of 5,000-20,000 m/z

a defined window width (in our case $25 \mathrm{kDa}$ ) rather than the differentiation of the entire spectrum as used in the standard Biotyper method. This is motivated by the fact that the noise has different intensities at different mass ranges. A maximum spectra normalization was used; in this approach the most intense peak from each spectrum is set to a value of 1 and the intensity of all other signals is calculated relative to this (22). The signal-to-noise threshold was set to 0.01 . Thus, in order to be assigned, a peak should have a relative intensity of at least $1 \%$ of the highest peak. By combining all these parameters, we were able to eliminate most of the noise and artefacts from raw spectra which otherwise resulted in unrealistic classification of the cell lines.

The MALDI mass spectra consist of a type of multivariate data, with each mass signal defining one molecular dimension, and the process of evaluating mass spectra requires multivariate statistical methods that grant the differentiation of samples (16). A multivariate statistical method used to analyze MALDI spectra is the PCA. The principle of this method is represented by the extraction of variance within a data set, reducing the dimensionality of the data set while retaining the information present in the original data set what leads to spectra with similar variation characteristics that can be clustered together and the differences between samples groups can be easily visualized in the system (16).

PCA analysis could be applied successfully on cancer cells by using their group spectra for the differentiation of pathology with a high sensitivity and selectivity (23). We began by performing PCA analysis using the whole mass range of
5,000-20,000 m/z. As in the MSP classification, this mass range proved to comprise too many groups of variables which contain redundant information and interfere with a realistic classification. By selecting the $\mathrm{m} / \mathrm{z}$ interval of 9,500-12,500, a minimized profile was created which retains more meaningful peaks, and at the same time eliminates peaks with similar behavior. The restricted mass range also decreased the number of contributory principle components to three (Fig. 7).

Since the PC3 contribution to the dataset variance is low, the experiment could disregard it and take only the PC1/PC2 two-dimensional clustering into consideration, thus reducing the dataset to an even lower dimensional space. The PC1/PC2 showed a closer clustering of the three human carcinomas and of the two murine melanomas. The human melanoma is plotted at a significant distance from both murine melanoma and human carcinoma groups (Fig. 5).

MALDI-imaging mass spectrometry (IMS) is also used with PCA analysis in a similar manner as it was used in our study (24). In MALDI-IMS, the PCA is used to classify cells in a single tissue section, while in our study it was used to classify different cell lines (25). The present approach can be used for cancer studies in combination with MALDI-IMS. The combined proteomic profiles from these two approaches can be compared revealing further information about tumor complexity.

PCA analysis has been used in conjunction with Raman spectroscopy in a cancer study by Bodanese et al (23) for the successful discrimination of basal cell carcinoma and melanoma from normal skin biopsies. Raman spectroscopy is a 
spectroscopic technique which typically uses electromagnetic radiation emitted by a sample when treated with a laser (26). The major difference from MALDI-TOF MS is that Raman microscopy does not use a laser to ablate the proteins from the target. Although Raman spectroscopy has proven to be a valuable technique, MALDI-TOF MS is a more widely established as a routine technique in many laboratories (27). It does not require complex sample preparation and requires minimal training, particularly after the technique was standardized and validated (5).

MSP are used as an identification basis by the MALDI Biotyper software. MSP are a reference spectra which contain one or several peak lists assigned to a bacteria species or strain. In the Biotyper identification workflow, one MSP should contain a certain number of peaks. This will provide an unambiguous identification of an unknown organism when its mass spectrum is compared to the reference MSP stored in a database. The software generates scoring values between 0 and 3 . The higher the score value, the higher the degree of similarity between the sample and the reference MSP. Apart from identification, the generated MSPs can be used for sample classification instead of raw spectra. We decided to use this system to compare the obtained scores to the PCA classification. In our case, the cumulated spectra (10 individual spectra of 10,000 laser shots) were used for creating two types of MSPs: individual MSPs which contain the peak list for one single cell line and three group MSPs which contained the cumulated peak list of the three main groups of cancers cells: human melanoma (A375), human carcinoma (MDA-MB-231, HepG2 and MCF7) and murine melanoma (B164A5 and B16-F0) cells. In this study, a combination of parameters was tested and the optimum was used for MSP creation. The use of the whole mass range of 5,000-20,000 m/z, did not provide a realistic classification according to the type of cancer and the originating species. Therefore, several mass ranges were selected, as well as different numbers of peaks to be included in the MSP. A number of ten peaks from the $9,500-12,500 \mathrm{~m} / \mathrm{z}$ interval proved to be more appropriate in providing realistic results.

When compared to the group MSPs, all cell lines were correctly attributed to their corresponding group (Table I). The differences between the first and second hit were substantial providing an unambiguous classification.

The MSP-based dendrogram (Fig. 9) revealed similar results to the PCA-based one, proving consistency between the two approaches. These results are in agreement with those described by Geiger et al (2012) in a previous study in the way that species relationship prevails to organ relationship, data obtained by using an LTQ-Orbitrap family mass spectrometer with a 'high field' Orbitrap mass analyzer (28). In the present study, the species-based relationship was stronger than the relationship between different types of cancer from which the cells originated, a fact that was revealed for the first time by the means of the MALDI-TOF MS technique. In addition, the restricted mass range of 9,500-12,500 $\mathrm{m} / \mathrm{z}$ was not previously reported.

The results revealed a closer relationship between HepG2 (liver carcinoma) and MDA-MB-231 (breast carcinoma) than between the two breast carcinomas cell lines. This can be explained by a high degree of similarity between the proteomic profiles of the different human cancer cell lines, a fact previ- ously reported (28). Another hypothesis for this similarity is the adaptation of the cells to in vitro culture which could lead to a clone selection based on their proliferative potential with the loss of the functions that are not vital (29). Although there were similarities between the HepG2 and MDA-MB-231 cell lines, the MSP-based scoring system revealed that the protein expression was clearly distinct between the cell lines.

The distance level between the MCF7 and MDA-MB-231 cell lines revealed by MSP analysis could be explained by the fact that, even though these cell lines have the same origin (pleural effusions of metastatic mammary patients), the MCF7 cell line (non-invasive cells) expresses specific markers for luminal epithelial phenotype and is employed as a model for estrogen receptor-positive tumors, whereas the MDA-MB-231 cell line (invasive cells) expresses mesenchymal markers (vimentin) and is used for estrogen receptor-negative breast cancers (30). Moreover, there were observed significant differences between the MCF7 and MDA-MB-231 proteomic profiles (31).

In our previous study, it was shown that there are differences between B16 murine melanoma cell sublines as regard molecular profile and proliferation behavior, B164A5 being described as a highly metastatic cell line (32). Moreover, the human melanoma cell line, A375, proved to be a valuable asset in inducing reproducible animal models with primary melanoma (33). The classification of melanoma cells in different clades could be explained by the fact that they originate from different species (human and murine), and in addition, A375 cells are an amelanotic primary melanoma cell line and their metastatic potential is lower compared to that of the murine melanoma cells, B164A5 and B16-F0 $(32,33)$.

The differences between the cancer cell lines tested observed by applying MALDI-TOF MS assert the data that were presented after microscopic evaluation in terms of shape and invasive capacity.

In conclusion, although different, the two statistical approaches (PCA and MSP) provided similar results. The MSP-based approach can be used for building groups which contain one cancer type or one cancer type from a given species. We were able to differentiate the cells according to cancer type (carcinoma versus melanoma) and the same cancer type originating from two different species (human versus mice melanomas). This proves that the MSP approach can be used not only to identify unknown samples, but also for their classification.

The results highlighted the necessity to establish minimized profiles comprising a restricted mass range. The MALDI-TOF acquisition method could also be improved to obtain maximum resolution for the newly establish mass range of 9,500-12,500 $\mathrm{m} / \mathrm{z}$. We do not exclude the possibility of other discriminative peaks outside this range. However, this could not be achieved using Biotyper software which does not allow direct peak list editing.

\section{Acknowledgements}

This study was financially supported by a grant of the Romanian National Authority for Scientific Research and Innovation, CNCS-UEFISCDI, project no. PN-II-RU-TE-2014-4-2842 (Dorina Coricovac). This study was performed within the 
Center of Genomic Medicine from the 'Victor Babes' University of Medicine and Pharmacy Timisoara, POSCCE Project ID: 1854, SMIS code: 48749, 'Center of Genomic Medicine v2', contract no. 677/09.04.2015.

\section{References}

1. Kallow W, Erhard M, Shah HN, Raptakis E and Welker M: MALDI-TOF MS for Microbial Identification: Years of Experimental Development to an Established Protocol. In: Mass Spectrometry for Microbial Proteomics. Shah HN and Gharbia SE (eds). John Wiley \& Sons, Ltd., Chichester, pp255-276, 2010.

2. Shah HN and Gharbia SE (eds): Mass Spectrometry for Microbial Proteomics. John Wiley and Sons Ltd., Chichester, UK, 2010.

3. Yang C, He Z and Yu W: Comparison of public peak detection algorithms for MALDI mass spectrometry data analysis. BMC Bioinformatics 10: 4, 2009.

4. Jarman KH, Daly DS, Anderson KK and Wahl KL: A new approach to automated peak detection. Chemom Intell Lab Syst 69: 61-76, 2003.

5. Singhal N, Kumar M, Kanaujia PK and Virdi JS: MALDI-TOF mass spectrometry: An emerging technology for microbial identification and diagnosis. Front Microbiol 6: 791, 2015.

6. Karger A, Bettin B, Lenk M and Mettenleiter TC: Rapid characterisation of cell cultures by matrix-assisted laser desorption/ionisation mass spectrometric typing. J Virol Methods 164: 116-121, 2010.

7. Zhang X, Scalf M, Berggren TW, Westphall MS and Smith LM: Identification of mammalian cell lines using MALDI-TOF and LC-ESI-MS/MS mass spectrometry. J Am Soc Mass Spectrom 17: 490-499, 2006.

8. Povey JF, O'Malley CJ, Root T, Martin EB, Montague GA, Feary M, Trim C, Lang DA, Alldread R, Racher AJ, et al: Rapid high-throughput characterisation, classification and selection of recombinant mammalian cell line phenotypes using intact cell MALDI-ToF mass spectrometry fingerprinting and PLS-DA modelling. J Biotechnol 184: 84-93, 2014.

9. Wang P, Gao Q, Suo Z, Munthe E, Solberg S, Ma L, Wang M, Westerdaal NA, Kvalheim G and Gaudernack G: Identification and characterization of cells with cancer stem cell properties in human primary lung cancer cell lines. PLoS One 8: e57020, 2013.

10. Dirks WG and Drexler HG: Authentication of Cancer Cell Lines by DNA Fingerprinting. In: Cancer Cell Culture: methods and Protocols. 1st edition. Langdon SP (ed). Humana Press, Totowa, NJ, pp33-42, 2004.

11. Greve B, Kelsch R, Spaniol K, Eich HT and Götte M: Flow cytometry in cancer stem cell analysis and separation. Cytometry A 81: 284-293, 2012.

12. Duraiyan J, Govindarajan R, Kaliyappan K and Palanisamy M: Applications of immunohistochemistry. J Pharm Bioallied Sci 4 (Suppl 2): S307-S309, 2012.

13. Coricovac DE, Moacă EA, Pinzaru I, Cîtu C, Soica C, Mihali CV, Păcurariu C, Tutelyan VA, Tsatsakis A and Dehelean CA: Biocompatible Colloidal Suspensions Based on Magnetic Iron Oxide Nanoparticles: Synthesis, Characterization and Toxicological Profile. Front Pharmacol 8: 154, 2017.

14. Hillenkamp F and Karas M: Mass spectrometry of peptides and proteins by matrix-assisted ultraviolet laser desorption/ionization. Methods Enzymol 193: 280-295, 1990.

15. Flatley B, Malone $P$ and Cramer R: MALDI mass spectrometry in prostate cancer biomarker discovery. Biochim Biophys Acta 1844: 940-949, 2014.

16. Cho YT, Chiang YY, Shiea J and Hou MF: Combining MALDI-TOF and molecular imaging with principal componen analysis for biomarker discovery and clinical diagnosis of cancer. Genomic Medicine, Biomarkers and Health Sciences 4: 3-6, 2012.

17. Rodrigo MA, Zitka O, Krizkova S, Moulick A, Adam V and Kizek R: MALDI-TOF MS as evolving cancer diagnostic tool: A review. J Pharm Biomed Anal 95: 245-255, 2014.
18. Nawarak J, Huang-Liu R, Kao SH, Liao HH, Sinchaikul S, Chen ST and Cheng SL: Proteomics analysis of A375 human malignant melanoma cells in response to arbutin treatment. Biochim Biophys Acta 1794: 159-167, 2009.

19. Heger Z, Rodrigo MA, Krizkova S, Zitka O, Beklova M, Kizek R and Adam V: Identification of estrogen receptor proteins in breast cancer cells using matrix-assisted laser desorption/ionization time of flight mass spectrometry (Review). Oncol Lett 7: 1341-1344, 2014

20. Corona G, De Lorenzo E, Elia C, Simula MP, Avellini C, Baccarani U, Lupo F, Tiribelli C, Colombatti A and Toffoli G: Differential proteomic analysis of hepatocellular carcinoma. Int J Oncol 36: 93-99, 2010.

21. Kriegsmann J, Kriegsmann M and Casadonte R: MALDI TOF imaging mass spectrometry in clinical pathology: A valuable tool for cancer diagnostics (Review). Int J Oncol 46: 893-906, 2015.

22. Delorme A, Sejnowski T and Makeig S: Enhanced detection of artifacts in EEG data using higher-order statistics and independent component analysis. Neuroimage 34: 1443-1449, 2007.

23. Bodanese B, Silveira FL, Zângaro RA, Pacheco MTT, Pasqualucci CA and Silveira L Jr: Discrimination of basal cell carcinoma and melanoma from normal skin biopsies in vitro through Raman spectroscopy and principal component analysis. Photomed Laser Surg 30: 381-387, 2012.

24. Alexandrov T: MALDI imaging mass spectrometry: Statistical data analysis and current computational challenges. BMC Bioinformatics 13 (Suppl 16): S11, 2012.

25. Norris JL and Caprioli RM: Imaging mass spectrometry: A new tool for pathology in a molecular age. Proteomics Clin Appl 7: 733-738, 2013

26. Bumbrah GS and Sharma RM: Raman spectroscopy - Basic principle, instrumentation and selected applications for the characterization of drugs of abuse. Egypt J Forensic Sci 6: 209-215, 2016.

27. Ghebremedhin M, Heitkamp R, Yesupriya S, Clay B and Crane NJ: Accurate and rapid differentiation of Acinetobacter baumannii strains by Raman spectroscopy: a comparative study. J Clin Microbiol 55: 2480-2490, 2017.

28. Geiger T, Wehner A, Schaab C, Cox J and Mann M: Comparative proteomic analysis of eleven common cell lines reveals ubiquitous but varying expression of most proteins. Mol Cell Proteomics 11: M111.014050, 2012.

29. Pan C, Kumar C, Bohl S, Klingmueller U and Mann M: Comparative proteomic phenotyping of cell lines and primary cells to assess preservation of cell type-specific functions. Mol Cell Proteomics 8: 443-450, 2009.

30. Mladkova J, Sanda M, Matouskova E and Selicharova I: Phenotyping breast cancer cell lines EM-G3, HCC1937, MCF7 and MDA-MB-231 using 2-D electrophoresis and affinity chromatography for glutathione-binding proteins. BMC Cancer 10: $449,2010$.

31. Nagaraja GM, Othman M, Fox BP, Alsaber R, Pellegrino CM, Zeng Y, Khanna R, Tamburini P, Swaroop A and Kandpal RP: Gene expression signatures and biomarkers of noninvasive and invasive breast cancer cells: Comprehensive profiles by representational difference analysis, microarrays and proteomics. Oncogene 25: 2328-2338, 2006.

32. Danciu C, Falamas A, Dehelean C, Soica C, Radeke H, Barbu-Tudoran L, Bojin F, Pînzaru SC and Munteanu MF: A characterization of four B16 murine melanoma cell sublines molecular fingerprint and proliferation behavior. Cancer Cell Int 13: 75, 2013.

33. Avram S, Coricovac DE, Pavel IZ, Pinzaru I, Ghiulai R, Baderca F, Soica C, Muntean D, Branisteanu DE, Spandidos DA, et al: Standardization of A375 human melanoma models on chicken embryo chorioallantoic membrane and Balb/c nude mice. Oncol Rep 38: 89-99, 2017. 